\title{
On the compact approximation property
}

\author{
by \\ Vegard Lima, Åsvald Lima and Olav NygaArd (Kristiansand)
}

\begin{abstract}
We show that a Banach space $X$ has the compact approximation property if and only if for every Banach space $Y$ and every weakly compact operator $T: Y \rightarrow X$, the space

$$
\mathfrak{E}=\{S \circ T: S \text { compact operator on } X\}
$$

is an ideal in $\mathfrak{F}=\operatorname{span}(\mathfrak{E},\{T\})$ if and only if for every Banach space $Y$ and every weakly compact operator $T: Y \rightarrow X$, there is a net $\left(S_{\gamma}\right)$ of compact operators on $X$ such that $\sup _{\gamma}\left\|S_{\gamma} T\right\| \leq\|T\|$ and $S_{\gamma} \rightarrow I_{X}$ in the strong operator topology. Similar results for dual spaces are also proved.
\end{abstract}

1. Introduction. A Banach space $X$ is said to have the compact approximation property if for every compact set $K$ in $X$ and every $\varepsilon>0$, there is a compact operator $S: X \rightarrow X$ such that $\|S x-x\| \leq \varepsilon$ for all $x \in K$. If these approximating compact operators can be chosen with $\|S\| \leq 1$, we say that $X$ has the metric compact approximation property. If the approximating operators can be chosen to have finite rank, then we say that $X$ has the approximation property.

Formally the approximation property is stronger than the compact approximation property, but it was only recently, in 1992, that Willis [19] showed that there is a separable reflexive Banach space with the compact approximation property but failing the approximation property.

Let $\mathfrak{E}$ be a closed subspace of a Banach space $\mathfrak{F}$. Then $\mathfrak{E}$ is an ideal in $\mathfrak{F}$ if its annihilator, $\mathfrak{E}^{\perp} \subseteq \mathfrak{F}^{*}$, is the kernel of a norm one projection on $\mathfrak{F}^{*}$. A linear operator $\phi: \mathfrak{E}^{*} \rightarrow \mathfrak{F}^{*}$ is called a Hahn-Banach extension operator if $\left(\phi e^{*}\right)(e)=e^{*}(e)$ and $\left\|\phi e^{*}\right\|=\left\|e^{*}\right\|$ for all $e \in \mathfrak{E}$ and $e^{*} \in \mathfrak{E}^{*}$. Denote the set of all Hahn-Banach extension operators $\phi: \mathfrak{E}^{*} \rightarrow \mathfrak{F}^{*}$ by $\mathrm{BB}(\mathfrak{E}, \mathfrak{F})$. Clearly, $\mathrm{B}(\mathfrak{E}, \mathfrak{F}) \neq \emptyset$ if and only if $\mathfrak{E}$ is an ideal in $\mathfrak{F}$. If $\mathfrak{E}$ is a subspace of a normed space $\mathfrak{F}$, then we say that $\mathfrak{E}$ is an ideal in $\mathfrak{F}$ if $\overline{\mathfrak{E}}$ is an ideal in $\overline{\mathfrak{F}}$.

2000 Mathematics Subject Classification: Primary 46B20, 46B28, 47L05.

Key words and phrases: compact approximation property, spaces of operators, operator ideals. 
Let $X$ and $Y$ be Banach spaces. We denote by $\mathcal{L}(Y, X)$ the Banach space of all bounded linear operators from $Y$ to $X$, and $\mathcal{F}(Y, X), \mathcal{K}(Y, X)$, and $\mathcal{W}(Y, X)$ denote the subspaces of finite rank, compact, and weakly compact operators respectively.

In [11, Theorem 5.1] Lima and Oja proved that a Banach space $X$ has the approximation property if and only if $\mathcal{F}(Y, X)$ is an ideal in $\mathcal{K}(Y, X)$ for all Banach spaces $Y$. Lima, Nygaard, and Oja extended this result in [10, Theorem 3.3] when they proved that $X$ has the approximation property if and only if $\mathcal{F}(Y, X)$ is an ideal in $\mathcal{W}(Y, X)$ for all Banach spaces $Y$. There exists a closed subspace $X$ of $\ell_{1}$ without the compact approximation property (cf. [18] or e.g. [16, p. 107]). For this space $X$ we have $\mathcal{K}(Y, X)=\mathcal{W}(Y, X)$ for all Banach spaces $Y$. This follows from the Schur property of $\ell_{1}$. In this paper we show that there is a result (see Theorem 2.2), somewhat analogous to Theorem 3.3 in [10], which characterizes the compact approximation property.

In Section 2, we show (see Theorem 2.2) that a Banach space has the compact approximation property if and only if for all Banach spaces $Y$ and all $T \in \mathcal{W}(Y, X)$, the space

$$
\mathfrak{E}=\{S \circ T: S \in \mathcal{K}(X, X)\}
$$

is an ideal in $\mathfrak{F}=\operatorname{span}(\mathfrak{E},\{T\})$. This result is applied in Theorem 2.3 to show that a Banach space $X$ has the compact approximation property if and only if for every Banach space $Y$ and every weakly compact operator $T: Y \rightarrow X$, there is a net $\left(S_{\gamma}\right)$ of compact operators on $X$ such that $\sup _{\gamma}\left\|S_{\gamma} T\right\| \leq\|T\|$ and $S_{\gamma} \rightarrow I_{X}$ in the strong operator topology.

In Section 3, we show that Theorems 2.2 and 2.3 can be used to give similar characterizations of the compact approximation property for dual spaces. In this case (see Theorem 3.2), $X$ is the domain space and not the range space as in Section 2. Moreover, in Section 3 we give a characterization of the compact approximation property with conjugate operators for the dual of a Banach space $X$ in terms of the approximability of weakly compact operators into $X^{* *}$ in the strong operator topology by certain compact operators.

In Theorem 3.8 we show that $X^{*}$ has the compact approximation property with conjugate operators if and only if $X^{*}$ has the compact approximation property and $\mathcal{K}(X, \widehat{X})$ is an ideal in $\mathcal{K}\left(X, \widehat{X}^{* *}\right)$ for every equivalent renorming $\widehat{X}$ of $X$.

Let us fix some notation. In a normed linear space $X$, we denote the closed unit ball by $B_{X}$. If $A$ is a subset of $X$, then $\bar{A}$ denotes the closure of $A, \operatorname{span}(A)$ denotes the linear span of $A$, and $\operatorname{conv}(A)$ is the convex hull of $A$. The identity operator on $X$ is denoted by $I_{X}$.

A left approximate identity in a normed algebra $\mathcal{A}$ is a net $\left(e_{i}\right)_{i \in I}$ in $\mathcal{A}$ such that $\lim _{i}\left\|e_{i} x-x\right\|=0$ for all $x \in \mathcal{A}$. In [4] Dixon showed that 
if a Banach space $X$ has the approximation property (resp. the compact approximation property), then $\overline{\mathcal{F}}(X, X)$ (resp. $\mathcal{K}(X, X)$ ) has a left approximate identity. If every compact subset of $X$ is contained in the closed unit range of some operator in $\overline{\mathcal{F}}(X, X)$ (resp. $\mathcal{K}(X, X))$, then the converse is true (see [4, Theorem 2.7]). In this note we repeatedly use a construction by Davis-Figiel-Johnson-Pełczyński [3] which enables us, for every compact subset $K$ of $X$, to find a reflexive Banach space $Z$ and an operator $J \in \mathcal{K}(Z, X)$ such that $K \subseteq J\left(B_{Z}\right)$.

2. The compact approximation property. In [15, Theorem 1.e.4] Lindenstrauss and Tzafriri give a list of five equivalent formulations of the approximation property for Banach spaces. We start by stating the analogous theorem characterizing spaces with the compact approximation property.

TheOrem 2.1. Let $X$ be a Banach space. The following statements are equivalent:

(i) $X$ has the compact approximation property.

(ii) For every Banach space $Y$, the space of compact operators $\mathcal{K}(Y, X)$ is dense in $\mathcal{L}(Y, X)$, in the topology $\tau$ of uniform convergence on compact sets.

(iii) For every Banach space $Y$, the space of compact operators $\mathcal{K}(X, Y)$ is dense in $\mathcal{L}(X, Y)$, in the topology $\tau$ of uniform convergence on compact sets.

(iv) For every choice of $\left\{x_{n}\right\}_{n=1}^{\infty} \subseteq X,\left\{x_{n}^{*}\right\}_{n=1}^{\infty} \subseteq X^{*}$ such that

$$
\sum_{n=1}^{\infty}\left\|x_{n}^{*}\right\|\left\|x_{n}\right\|<\infty \quad \text { and } \quad \sum_{n=1}^{\infty} x_{n}^{*}\left(S x_{n}\right)=0
$$

for all $S \in \mathcal{K}(X, X)$, we have $\sum_{n=1}^{\infty} x_{n}^{*}\left(x_{n}\right)=0$.

(v) For every Banach space $Y$, every compact operator $T \in \mathcal{K}(Y, X)$, and every $\varepsilon>0$, there is a compact operator $S \in \mathcal{K}(X, X)$ with $\|T-S T\|<\varepsilon$.

(vi) For every Banach space $Y$, every finite-dimensional subspace $G \subseteq$ $\mathcal{K}(Y, X)$, and every $\varepsilon>0$, there is a compact operator $S \in \mathcal{K}(X, X)$ with $\|T-S T\|<\varepsilon\|T\|$ for all $T \in G$.

Proof. For the equivalence of (i) through (iv), see the proof of Theorem 1.e.4 in [15].

(i) $\Rightarrow$ (vi). Let $K=\overline{\operatorname{conv}}\left\{T y: y \in B_{Y}, T \in B_{G}\right\}$ and let $\varepsilon>0$. Since $K$ is compact, by (i) there is an operator $S \in \mathcal{K}(X, X)$ with $\|S x-x\|<\varepsilon$ for all $x \in K$. From this it follows that $\|S T-T\|<\varepsilon\|T\|$ for all $T \in G$.

$(\mathrm{vi}) \Rightarrow(\mathrm{v})$ is trivial. 
$(\mathrm{v}) \Rightarrow(\mathrm{iv})$. Let $\left\{x_{n}\right\}_{n=1}^{\infty} \subseteq X$ and $\left\{x_{n}^{*}\right\}_{n=1}^{\infty} \subseteq X^{*}$ with $\sum_{n=1}^{\infty}\left\|x_{n}^{*}\right\|\left\|x_{n}\right\|$ $<\infty$ and $\sum_{n=1}^{\infty} x_{n}^{*}\left(S x_{n}\right)=0$ for all $S \in \mathcal{K}(X, X)$. We may assume that $\sum_{n=1}^{\infty}\left\|x_{n}^{*}\right\|<\infty$ and $1 \geq\left\|x_{n}\right\| \rightarrow 0$. Let $K=\overline{\operatorname{conv}}\left( \pm x_{n}\right)$. Then $K$ is a compact subset of $B_{X}$.

By the Davis-Figiel-Johnson-Pełczyński factorization procedure [3], there is a reflexive Banach space $Z$, a compact operator $J \in \mathcal{K}(Z, X)$, and a sequence $\left\{z_{n}\right\}_{n=1}^{\infty} \subseteq B_{Z}$ such that $J z_{n}=x_{n}$. For $S \in \mathcal{K}(X, X)$ we get

$$
0=\sum_{n=1}^{\infty} x_{n}^{*}\left(S x_{n}\right)=\left(\sum_{n=1}^{\infty} x_{n}^{*} \otimes z_{n}\right)(S J) .
$$

The functional $\phi=\sum_{n=1}^{\infty} x_{n}^{*} \otimes z_{n} \in \mathcal{K}(Z, X)^{*}$ therefore annihilates the subspace $\mathfrak{E}=\{S \circ J: S \in \mathcal{K}(X, X)\}$ of $\mathcal{K}(Z, X)$. By $(\mathrm{v})$ we have $J \in \overline{\mathfrak{E}}$, so from the continuity of $\phi$ it follows that $0=\phi(J)=\sum_{n=1}^{\infty} x_{n}^{*}\left(x_{n}\right)$.

When we compare Theorem 2.1 with Theorem 1.e.4 in [15], we see that they are almost identical. There is only a "minor" difference in statement (v). Namely, $S T$ in (v) above is written as $T_{1} \in \mathcal{F}(Y, X)$ in (v) of Theorem 1.e.4. This apparently minor difference turns out to be very important.

In [10, Theorem 3.3] Lima, Nygaard, and Oja proved that a Banach space $X$ has the approximation property if and only if $\mathcal{F}(Y, X)$ is an ideal in $\mathcal{W}(Y, X)$ for all Banach spaces $Y$. They also showed that $\mathcal{K}(Y, X)$ being an ideal in $\mathcal{W}(Y, X)$ for all Banach spaces $Y$ is a weaker property than the compact approximation property. The next theorem gives a characterization of the compact approximation property by ideals.

THEOREM 2.2. Let $X$ be a Banach space. The following statements are equivalent:

(i) $X$ has the compact approximation property.

(ii) For every Banach space $Y$ and every $T \in \mathcal{W}(Y, X)$,

$$
\mathfrak{E}=\{S \circ T: S \in \mathcal{K}(X, X)\}
$$

is an ideal in $\mathfrak{F}=\operatorname{span}(\mathfrak{E},\{T\})$.

(iii) Same as (ii), but with $T \in \mathcal{K}(Y, X)$.

Proof. (i) $\Rightarrow$ (ii). Assume first that $Y$ is reflexive, and let $T \in \mathcal{W}(Y, X)$.

Let $\left(S_{\gamma}\right)$ be a net in $\mathcal{K}(X, X)$ such that $S_{\gamma} \rightarrow I_{X}$ uniformly on compact sets. Let $v \in \mathfrak{E}^{*}$ and $\varepsilon>0$. Since $\mathfrak{E} \subseteq \mathcal{K}(Y, X)$, by a theorem of Feder and Saphar [6, Theorem 1], we can write $v=\sum_{n=1}^{\infty} x_{n}^{*} \otimes y_{n}$ where $\left\{x_{n}^{*}\right\}_{n=1}^{\infty} \subseteq X^{*}$, $\left\{y_{n}\right\}_{n=1}^{\infty} \subseteq Y$, and $\sum_{n=1}^{\infty}\left\|x_{n}^{*}\right\|\left\|y_{n}\right\|<\|v\|+\varepsilon$. We may assume $\sum_{n=1}^{\infty}\left\|x_{n}^{*}\right\|$ $<\infty$ and $\left\|y_{n}\right\| \rightarrow 0$. Then $\left\{T y_{n}\right\}_{n=1}^{\infty}$ is a relatively compact subset of $X$, and hence 


$$
\begin{aligned}
& \left|\sum_{n=1}^{\infty} x_{n}^{*}\left(T y_{n}\right)-\sum_{n=1}^{\infty} x_{n}^{*}\left(S_{\gamma} T y_{n}\right)\right| \\
& \leq\left(\sum_{n=1}^{\infty}\left\|x_{n}^{*}\right\|\right) \underset{n}{\left(\sup _{n}\left\|\left(I_{X}-S S_{\gamma}\right) T y_{n}\right\|\right) \underset{\gamma}{\rightarrow} 0 .}
\end{aligned}
$$

Thus we can define a linear operator $\Phi: \mathfrak{E}^{*} \rightarrow \mathfrak{F}^{*}$ by

$$
(\Phi v)(T)=\sum_{n=1}^{\infty} x_{n}^{*}\left(T y_{n}\right)
$$

(Note that this definition of $\Phi v$ is independent of the particular representation $\sum_{n=1}^{\infty} x_{n}^{*} \otimes y_{n}$ of $v$.) Since $\sum_{n=1}^{\infty}\left\|x_{n}^{*}\right\|\left\|y_{n}\right\|<\|v\|+\varepsilon$ and $\varepsilon>0$ is arbitrary, $\Phi$ must have norm one, which is to say that $\Phi$ is a Hahn-Banach extension operator.

Now let $Y$ be an arbitrary Banach space and let $T \in \mathcal{W}(Y, X)$. We may assume that $\|T\|=1$. By $[10$, Theorem 2.3] there is a reflexive Banach space $Z$ and a norm one operator $J: Z \rightarrow X$ with $Z \subseteq X$ (as sets) such that $T$ can be factorized through $Z$ with $J$. That is, we can write $T=J \circ \widehat{T}$, where $\widehat{T}: Y \rightarrow Z$ is defined by $\widehat{T} y=T y \in Z \subseteq X$.

By the first part of the proof $\mathfrak{E}_{J}=\{S \circ J: S \in \mathcal{K}(X, X)\}$ is an ideal in $\mathfrak{F}_{J}=\operatorname{span}\left(\mathfrak{E}_{J},\{J\}\right)$. Let $\mathfrak{E}=\{S \circ T: S \in \mathcal{K}(X, X)\}, \mathfrak{F}=\operatorname{span}(\mathfrak{E},\{T\})$, and let $G \subseteq \mathfrak{F}$ be finite-dimensional. There is a finite-dimensional subspace $H \subseteq$ $\mathfrak{F}_{J}$ such that $G=\{U \circ \widehat{T}: U \in H\}$. Let $\varepsilon>0$. By the local characterization of ideals (see e.g. [5], [8], or [9]), there is a linear operator $V_{H}: H \rightarrow \mathfrak{E}_{J}$ such that $\left\|V_{H}\right\| \leq 1+\varepsilon$ and such that $V_{H}(S \circ J)=S \circ J$ when $S \circ J \in H$ and $S \in \mathcal{K}(X, X)$.

Define $V_{G}: G \rightarrow \mathfrak{E}$ by

$$
V_{G}(U)=V_{H}(U) \circ \widehat{T}
$$

Then $\left\|V_{G}\right\| \leq\left\|V_{H}\right\| \leq 1+\varepsilon$ and if $S \in \mathcal{K}(X, X)$ with $S \circ T \in G$, then $S \circ J \in H$ and $V_{G}(S \circ J)=S \circ T$. From the local characterization of ideals it follows that $\mathfrak{E}$ is an ideal in $\mathfrak{F}$.

(ii) $\Rightarrow$ (iii) is trivial; let us prove (iii) $\Rightarrow$ (i). Let $K \subseteq B_{X}$ be compact and let $\varepsilon>0$. Using Lemma 1.1 in [10] we find a separable reflexive Banach space $Z$ and a compact operator $J: Z \rightarrow X$ with $\|J\| \leq 1$ such that $K \subseteq J\left(B_{Z}\right) \subseteq B_{X}$. Note that since $J^{*} X^{*}$ is norm-dense in $Z^{*}$ we have

$$
\mathcal{F}(Z, X) \subseteq \overline{\{S \circ J: S \in \mathcal{F}(X, X)\}} .
$$

Letting $Z$ be the Banach space and $J$ be the operator in (iii) we use Lemma 1.4 in [10] to find a net $\left(S_{\gamma} J\right)$ such that $\sup _{\gamma}\left\|S_{\gamma} J\right\| \leq\|J\| \leq 1$ and $S_{\gamma} J \rightarrow J$ in the strong operator topology. 
By construction $K$ is also compact in $Z$ (see [10, Lemma 2.1]). Thus $S_{\gamma} J \rightarrow J$ uniformly on $K$, so for large $\gamma$,

$$
\sup _{x \in K}\left\|x-S_{\gamma} x\right\|=\sup _{z \in K}\left\|J z-S_{\gamma} J z\right\|<\varepsilon .
$$

Note that we cannot control the norms of the $S_{\gamma}$ 's.

Lima and Oja have given a characterization of the approximation property [13, Theorem 3.1] in terms of approximability of operators in the strong operator topology. The next result is a similar theorem characterizing the compact approximation property.

TheOrem 2.3. Let $X$ be a Banach space. The following statements are equivalent:

(i) $X$ has the compact approximation property.

(ii) For every Banach space $Y$, and every finite-dimensional subspace $G \subseteq \mathcal{W}(Y, X)$, there is a net $\left(S_{\gamma}\right) \subseteq \mathcal{K}(X, X)$ with $\sup _{\gamma}\left\|S_{\gamma} T\right\| \leq\|T\|$ for all $T \in G$, and such that $S_{\gamma} \rightarrow I_{X}$ in the strong operator topology.

(iii) For every Banach space $Y$, and every finite-dimensional subspace $G \subseteq \mathcal{W}(Y, X)$, there is a net $\left(S_{\gamma}\right) \subseteq \mathcal{K}(X, X)$ such that for all $T \in G$, $\sup _{\gamma}\left\|S_{\gamma} T\right\| \leq\|T\|$ and $T^{*} S_{\gamma}^{*} \rightarrow T^{*}$ in the strong operator topology.

(iv) For every separable reflexive Banach space $Y$ and every $T \in \mathcal{K}(Y, X)$, there is a net $\left(S_{\gamma}\right) \subseteq \mathcal{K}(X, X)$ with $\sup _{\gamma}\left\|S_{\gamma} T\right\| \leq\|T\|$ such that $S_{\gamma} T \rightarrow T$ in the strong operator topology.

Proof. (i) $\Rightarrow$ (ii) and (i) $\Rightarrow$ (iii). Let $Y$ be a Banach space, and let $G \subseteq$ $\mathcal{W}(Y, X)$ be a finite-dimensional subspace. Let

$$
K=\overline{\operatorname{conv}}\left\{T y: y \in B_{Y}, T \in B_{G}\right\} .
$$

Then $K$ is a weakly compact subset of $X$. In order to find a net that converges strongly to the identity on all of $X$ we will work with weakly compact sets larger than $K$. Let

$$
\Gamma=\left\{(E, F): E \subseteq X, \operatorname{dim} E<\infty, F \subseteq X^{*}, \operatorname{dim} F<\infty\right\}
$$

and order $\Gamma$ by inclusion. For each $\gamma=(E, F) \in \Gamma$, we define

$$
K_{\gamma}=\overline{\operatorname{conv}}\left(K \cup B_{E}\right) \text {. }
$$

Since $K_{\gamma}$ is convex and weakly compact, there exist, by Lemma 1.1 in [10] (see also Theorem 2.3 in [10]), a reflexive Banach space $Z_{\gamma}$, a weakly compact norm one operator $J_{\gamma}: Z_{\gamma} \rightarrow X$, and a linear isometry $\Psi_{\gamma}: G \rightarrow \mathcal{W}\left(Y, Z_{\gamma}\right)$ such that $T=J_{\gamma} \circ \Psi_{\gamma}(T)$ for all $T \in G$.

Let $\mathfrak{E}_{\gamma}=\left\{S \circ J_{\gamma}: S \in \mathcal{K}(X, X)\right\}$ and let $\mathfrak{F}_{\gamma}=\operatorname{span}\left(\mathfrak{E}_{\gamma}, J_{\gamma}\right)$. Since $J_{\gamma}^{* *}$ is injective (see $[3$, Lemma 1$]$ ), so that $J_{\gamma}^{*} X^{*}$ is norm dense in $Z_{\gamma}^{*}$, it follows that

$$
\mathcal{F}(Z, X) \subseteq \overline{\mathfrak{E}}_{\gamma} \subseteq \mathcal{K}(Z, X)
$$


By Theorem 2.2, $\overline{\mathfrak{E}}_{\gamma}$ is an ideal in $\overline{\mathfrak{F}}_{\gamma}$. By Lemma 1.4 in [10], for each $\gamma$, there is a net $\left(S_{\alpha}\right)$ in $\mathcal{K}(X, X)$ such that $\sup _{\alpha}\left\|S_{\alpha} J_{\gamma}\right\| \leq\left\|J_{\gamma}\right\| \leq 1$ and $S_{\alpha} J_{\gamma} \rightarrow_{\alpha} J_{\gamma}$ in the strong operator topology. It follows that

$$
x^{*}\left(S_{\alpha} J_{\gamma} z\right) \underset{\alpha}{\rightarrow} x^{*}\left(J_{\gamma} z\right)
$$

for all $z \in Z_{\gamma}$ and all $x^{*} \in X^{*}$.

For each $\gamma=(E, F)$, we have $B_{Z_{\gamma}} \subseteq K_{\gamma}$. Since $S_{E}$ and $S_{F}$ are compact, we can choose $\alpha$ such that

$$
\left|x^{*}\left(S_{\alpha} x\right)-x^{*}(x)\right| \leq \frac{\|x\|\left\|x^{*}\right\|}{\operatorname{dim} E+\operatorname{dim} F}
$$

for all $x \in E$ and all $x^{*} \in F$. Let us denote this $S_{\alpha}$ by $S_{\gamma}$. Then $\left(S_{\gamma}\right)_{\gamma \in \Gamma}$ is a net in $\mathcal{K}(X, X)$ and $S_{\gamma} \rightarrow I_{X}$ in the weak operator topology. By taking convex combinations, if necessary, we may assume that $S_{\gamma} \rightarrow I_{X}$ in the strong operator topology. By construction

$$
\sup _{\gamma}\left\|S_{\gamma} T\right\|=\sup _{\gamma}\left\|S_{\gamma} J_{\gamma} \Psi_{\gamma}(T)\right\| \leq\left\|\Psi_{\gamma}(T)\right\|=\|T\|
$$

for all $T \in G$. Hence (ii) is proved.

To finish the proof of (iii), note that $\Psi_{\gamma}(T)(y)=T y$ for all $\gamma$ and all $T \in G$. If $T \in G, x^{*} \in X^{*}$ and $y^{* *} \in Y^{* *}$, then $z=T^{* *} y^{* *}=\Psi_{\gamma}(T)^{* *} y^{* *}$ $\in Z_{\gamma}$, and

$$
y^{* *}\left(T^{*} x^{*}\right)-y^{* *}\left(T^{*} S_{\gamma}^{*} x^{*}\right)=x^{*}\left(J_{\gamma} z\right)-x^{*}\left(S_{\gamma} J_{\gamma} z\right) .
$$

With $\gamma=(E, F), x^{*} \in F$, and $T^{* *} y^{* *} \in E$, we get $T^{* *} y^{* *}=z=J_{\gamma} z$ and

$$
\left|y^{* *}\left(T^{*} x^{*}\right)-y^{* *}\left(T^{*} S_{\gamma}^{*} x^{*}\right)\right|=\left|x^{*}(z)-x^{*}\left(S_{\gamma} z\right)\right| \leq \frac{\left\|T^{* *} y^{* *}\right\|\left\|x^{*}\right\|}{\operatorname{dim} E+\operatorname{dim} F} .
$$

Thus $T^{*} S_{\gamma}^{*} \rightarrow T^{*}$ in the weak operator topology. By taking convex combinations we may assume that $T^{*} S_{\gamma}^{*} \rightarrow T^{*}$ in the strong operator topology.

The implications (iii) $\Rightarrow$ (ii) $\Rightarrow$ (iv) are easy, and (iv) $\Rightarrow$ (i) follows as in (iii) $\Rightarrow$ (i) of Theorem 2.2 .

Remark 2.1. Cho and Johnson proved in [2] that if $X$ is a reflexive Banach space with the compact approximation property, then $X$ has the metric compact approximation property. This result easily follows from Theorem 2.3. Just take $T=I_{X}$, which is weakly compact, and use (ii) of Theorem 2.3 to produce norm one approximating compact operators.

REMARK 2.2. Let $\mathcal{K}_{X}$ be the family of all compact absolutely convex subsets of $B_{X}$. For each $K \in \mathcal{K}_{X}$, let $Z_{K}$ be the reflexive space constructed from $K$ by the isometric version of the Davis-Figiel-Johnson-Pełczyński procedure in [10], and let $J_{K}: Z_{K} \rightarrow X$ be the associated compact norm one inclusion map. Let 


$$
\mathcal{F}_{K}=\left\{S \circ J_{K}: S \in \mathcal{F}(X, X)\right\} \quad \text { and } \quad \mathcal{K}_{K}=\left\{S \circ J_{K}: S \in \mathcal{K}(X, X)\right\} .
$$

The following are equivalent (see [10, Theorem 1.2]):

(1) $X$ has the approximation property.

(2) $J_{K} \in \overline{\mathcal{F}}_{K}$ for every $K \in \mathcal{K}_{X}$.

From Theorem 2.3 we see that the following are equivalent:

(1) $X$ has the compact approximation property.

(2) $J_{K} \in \overline{\mathcal{K}}_{K}$ for every $K \in \mathcal{K}_{X}$.

We have already noted that $J_{K}^{*} X^{*}$ is norm dense in $Z_{K}^{*}$, so $\mathcal{F}_{K}$ is always norm dense in $\mathcal{F}\left(Z_{K}, X\right)$. However, since $J_{K}$ is a compact operator, it follows that if $X$ does not have the compact approximation property, then, for some $K \in \mathcal{K}_{X}, \mathcal{K}_{K}$ is not norm dense in $\mathcal{K}\left(Z_{K}, X\right)$.

3. The compact approximation property for dual spaces. In this section we start by considering the compact approximation property for dual spaces and state theorems similar to Theorems 2.1-2.3. Recall that if $X$ is a Banach space such that $X^{*}$ has the approximation property then $X$ must also have the approximation property (see [15, Theorem 1.e.7]). The key to the proof of this theorem is that the principle of local reflexivity entails that the approximating operators can be chosen to be weak*-continuous. With the compact approximation property we are not so lucky, and it is unknown whether or not the compact approximation property for $X^{*}$ implies the compact approximation property for $X$. We also study a special version of the compact approximation property for dual spaces called the compact approximation property with conjugate operators, and we conclude this section with a comparison of these two approximation properties.

Theorem 3.1. Let $X$ be a Banach space. The following statements are equivalent:

(i) $X^{*}$ has the compact approximation property.

(ii) For every choice of $\left\{x_{n}^{*}\right\}_{n=1}^{\infty} \subseteq X^{*},\left\{x_{n}^{* *}\right\}_{n=1}^{\infty} \subseteq X^{* *}$ such that

$$
\sum_{n=1}^{\infty}\left\|x_{n}^{* *}\right\|\left\|x_{n}^{*}\right\|<\infty \quad \text { and } \quad \sum_{n=1}^{\infty} x_{n}^{* *}\left(S x_{n}^{*}\right)=0
$$

for all $S \in \mathcal{K}\left(X^{*}, X^{*}\right)$, we have $\sum_{n=1}^{\infty} x_{n}^{* *}\left(x_{n}^{*}\right)=0$.

(iii) For every Banach space $Y$, for every operator $T \in \mathcal{K}(X, Y)$, and for every $\varepsilon>0$ there is a compact operator $S \in \mathcal{K}\left(X, X^{* *}\right)$ with $\left\|T-T^{* *} S\right\|<\varepsilon$.

Proof. (i) $\Leftrightarrow$ (ii) and (i) $\Rightarrow$ (iii) follow from Theorem 2.1.

(iii) $\Rightarrow\left(\right.$ ii). Recall that $\mathcal{K}(X, Z)$ is isometrically isomorphic to $\mathcal{K}\left(Z^{*}, X^{*}\right)$ for $Z$ reflexive, and then argue as in (v) $\Rightarrow$ (iv) of Theorem 2.1.

Theorems 2.2 and 2.3 for dual spaces give the following result. 
THEOREM 3.2. Let $X$ be a Banach space. The following statements are equivalent:

(i) $X^{*}$ has the compact approximation property.

(ii) For every Banach space $Y$ and every $T \in \mathcal{W}(X, Y)$,

$$
\mathfrak{E}=\left\{T^{* *} \circ S: S \in \mathcal{K}\left(X, X^{* *}\right)\right\}
$$

is an ideal in $\mathfrak{F}=\operatorname{span}(\mathfrak{E},\{T\})$.

(iii) Same as (ii), but with $T \in \mathcal{K}(X, Y)$.

(iv) For every Banach space $Y$, and every finite-dimensional subspace $G \subseteq \mathcal{W}(X, Y)$, there is a net $\left(S_{\gamma}\right) \subseteq \mathcal{K}\left(X^{*}, X^{*}\right)$ such that for all $T \in G$, $\sup _{\gamma}\left\|T^{* *} S_{\gamma}^{*}\right\| \leq\|T\|$ and $T^{* *} S_{\gamma}^{*} \rightarrow T^{* *}$ in the strong operator topology.

(v) For every separable reflexive Banach space $Y$ and every $T \in \mathcal{K}(X, Y)$, there is a net $\left(S_{\gamma}\right) \subseteq \mathcal{K}\left(X^{*}, X^{*}\right)$ with $\sup _{\gamma}\left\|T^{* *} S_{\gamma}^{*}\right\| \leq\|T\|$ such that $S_{\gamma} T^{*}$ $\rightarrow T^{*}$ in the strong operator topology.

Proof. (i) $\Rightarrow$ (ii) for reflexive $Y$ follows from Theorem 2.2. For general $Y$ we use factorization through a reflexive Banach space as in Lemma 4.1 in [12]. We omit the details.

(ii) $\Rightarrow$ (iii) is trivial, and (iii) $\Rightarrow$ (i) follows as in Theorem 2.2.

(i) $\Rightarrow$ (iv) follows from (iii) of Theorem 2.3 by using the factorization in Lemma 4.1 of [12].

(iv) $\Rightarrow(\mathrm{v})$ is trivial, and $(\mathrm{v}) \Rightarrow(\mathrm{i})$ follows from the isometry $\mathcal{K}\left(Y, X^{*}\right) \cong$ $\mathcal{K}\left(X, Y^{*}\right)$ and $(\mathrm{iv}) \Rightarrow(\mathrm{i})$ of Theorem 2.3 .

We say that $X^{*}$ has the compact approximation property with conjugate operators if for every compact set $K$ in $X^{*}$ and every $\varepsilon>0$, there is a compact operator $S \in \mathcal{K}(X, X)$ such that $\left\|x^{*}-S^{*} x^{*}\right\|<\varepsilon$ for all $x^{*} \in K$.

THEOREM 3.3. Let $X$ be a Banach space. The following statements are equivalent:

(i) $X^{*}$ has the compact approximation property with conjugate operators.

(ii) For every choice $\left\{x_{n}^{*}\right\}_{n=1}^{\infty} \subseteq X^{*},\left\{x_{n}^{* *}\right\}_{n=1}^{\infty} \subseteq X^{* *}$ such that

$$
\sum_{n=1}^{\infty}\left\|x_{n}^{* *}\right\|\left\|x_{n}^{*}\right\|<\infty \quad \text { and } \quad \sum_{n=1}^{\infty} x_{n}^{* *}\left(S^{*} x_{n}^{*}\right)=0
$$

for all $S \in \mathcal{K}(X, X)$, we have $\sum_{n=1}^{\infty} x_{n}^{* *}\left(x_{n}^{*}\right)=0$.

(iii) For every Banach space $Y$, for every operator $T \in \mathcal{K}(X, Y)$, and for every $\varepsilon>0$ there is a compact operator $S \in \mathcal{K}(X, X)$ with $\|T-T S\|<\varepsilon$.

We omit the proof since it is similar to that of Theorem 3.1.

Theorems 2.2 and 2.3 have versions characterizing the compact approximation property with conjugate operators. 
Theorem 3.4. Let $X$ be a Banach space. The following statements are equivalent:

(i) $X^{*}$ has the compact approximation property with conjugate operators.

(ii) For every Banach space $Y$ and every $T \in \mathcal{W}(X, Y)$,

$$
\mathfrak{E}=\{T \circ S: S \in \mathcal{K}(X, X)\}
$$

is an ideal in $\mathfrak{F}=\operatorname{span}(\mathfrak{E},\{T\})$.

(iii) Same as (ii), but with $T \in \mathcal{K}(X, Y)$.

(iv) For every Banach space $Y$, and every finite-dimensional subspace $G \subseteq \mathcal{W}(X, Y)$, there is a net $\left(S_{\gamma}\right) \subseteq \mathcal{K}(X, X)$ such that for all $T \in G$, $\sup _{\gamma}\left\|T S_{\gamma}\right\| \leq\|T\|$ and $S_{\gamma}^{*} T^{*} \rightarrow T^{*}$ in the strong operator topology.

(v) For every separable reflexive Banach space $Y$ and every $T \in \mathcal{K}(X, Y)$, there is a net $\left(S_{\gamma}\right) \subseteq \mathcal{K}(X, X)$ with $\sup _{\gamma}\left\|T S_{\gamma}\right\| \leq\|T\|$ such that $S_{\gamma}^{*} T^{*} \rightarrow T^{*}$ in the strong operator topology.

We omit the proof since it is similar to the proof of Theorem 3.2.

In Section 2 we looked at operators with range in $X$, and in the first part of this section we have looked at operators with domain space $X$. We shall now look at operators with range in $X^{* *}$.

Lemma 3.5. Let $X$ and $Y$ be Banach spaces, and let $T \in \mathcal{W}\left(Y, X^{* *}\right)$. Let

$$
\mathfrak{E}=\left\{S^{* *} \circ T: S \in \mathcal{K}(X, X)\right\}
$$

and $\mathfrak{F}=\operatorname{span}(\mathfrak{E},\{T\})$. If $X^{*}$ has the compact approximation property with conjugate operators, then $\mathfrak{E}$ is an ideal in $\mathfrak{F}$. Moreover, if $Y$ is reflexive, then there is a Hahn-Banach extension operator $\Phi: \mathfrak{E}^{*} \rightarrow \mathfrak{F}^{*}$ such that $\Phi\left(x^{*} \otimes y\right)=x^{*} \otimes y$ for all $x^{*} \in X^{*}$ and all $y \in Y$.

Proof. The proof is similar to that of (i) $\Rightarrow($ ii) in Theorem 2.2. Here we start with $Y$ reflexive and with a net $\left(S_{\gamma}\right) \subseteq \mathcal{K}(X, X)$ such that $S_{\gamma}^{*} \rightarrow I_{X^{*}}$ uniformly on compact sets. Note that $\mathfrak{E} \subseteq \mathcal{K}(Y, X)$ and that $\mathfrak{F} \subseteq \mathcal{K}\left(Y, X^{* *}\right)$. The proof for general $Y$ is then as in Theorem 2.2.

REMARK 3.1. In Lemma 3.5 we showed that if $X^{*}$ has the compact approximation property with conjugate operators, then for all Banach spaces $Y$ and all operators $T \in \mathcal{K}\left(Y, X^{* *}\right)$,

$$
\mathfrak{E}=\left\{S^{* *} \circ T: S \in \mathcal{K}(X, X)\right\}
$$

is an ideal in $\mathfrak{F}=\operatorname{span}(\mathfrak{E},\{T\})$.

We have not been able to show that this ideal property characterizes the compact approximation property with conjugate operators for dual spaces $X^{*}$. 
However, we are able to show the following. Let us assume that

$$
\widehat{\mathfrak{E}}=\left\{S^{* *} \circ T: S \in \mathcal{K}(\widehat{X}, \widehat{X})\right\}
$$

is an ideal in $\widehat{\mathfrak{F}}=\operatorname{span}(\widehat{\mathfrak{E}},\{T\})$, for all Banach spaces $Y$, all operators $T \in \mathcal{K}\left(Y, \widehat{X}^{* *}\right)$, and all equivalent renormings $\widehat{X}$ of $X$. (Note that from Lemma 3.5 this is true whenever $X^{*}$ has the compact approximation property with conjugate operators.) Using the results of Section 4 in [14] we find a Hahn-Banach extension operator $\Psi: \mathfrak{E}^{*} \rightarrow \mathfrak{F}^{*}$ such that

$$
\Psi\left(x^{*} \otimes y^{* *}\right)(U)=\left(x^{*} \otimes y^{* *}\right)(U)
$$

for all $U \in \mathfrak{F}$ whenever $\mathfrak{E}$ contains the finite rank operators. In particular, $\mathfrak{E}$ contains the finite rank operators whenever $T^{* *}$ is injective.

Using the weak ${ }^{*}$ topology in $\mathfrak{F}^{* *}$, as in Lemma 1.4 of [10], we find a net $\left(S_{\gamma}^{* *} T\right)$ in $\mathbb{E}$ with $\sup _{\gamma}\left\|S_{\gamma}^{* *} T\right\| \leq\|T\|$ such that $\left.T^{*} S_{\gamma}^{*} \rightarrow T^{*}\right|_{X^{*}}$ in the strong operator topology. Theorem 3.6 below will show that this implies the compact approximation property with conjugate operators for $X^{*}$.

In [14, Theorem 4.5] Lima proved that $X^{*}$ has the approximation property if and only if $\mathcal{F}(Y, \widehat{X})$ is an ideal in $\mathcal{K}\left(Y, \widehat{X}^{* *}\right)$ for all Banach spaces $Y$ and all equivalent renormings $\widehat{X}$ of $X$.

In [13, Theorem 3.2] Lima and Oja gave a characterization of dual spaces with the approximation property by approximation of weakly compact operators by finite rank operators in the strong operator topology. Our next result shows that there is a similar characterization of the compact approximation property with compact operators, where we approximate weakly compact operators with certain composite compact operators in the strong operator topology.

Theorem 3.6. Let $X$ be a Banach space. The following statements are equivalent:

(i) $X^{*}$ has the compact approximation property with conjugate operators.

(ii) For every Banach space $Y$, and every finite-dimensional subspace $G \subseteq \mathcal{W}\left(Y, X^{* *}\right)$, there is a net $\left(S_{\gamma}\right) \subseteq \mathcal{K}(X, X)$ such that $\sup _{\gamma}\left\|S_{\gamma}^{* *} T\right\| \leq\|T\|$ for all $T \in G$ and $S_{\gamma}^{*} \rightarrow I_{X^{*}}$ in the strong operator topology.

(iii) For every Banach space $Y$, and every finite-dimensional subspace $G \subseteq \mathcal{W}\left(Y, X^{* *}\right)$, there is a net $\left(S_{\gamma}\right) \subseteq \mathcal{K}(X, X)$ such that for all $T \in G$, $\sup _{\gamma}\left\|S_{\gamma}^{* *} T\right\| \leq\|T\|$ and $\left.T^{*} S_{\gamma}^{*} \rightarrow T^{*}\right|_{X^{*}}$ in the strong operator topology.

(iv) For every separable reflexive Banach space $Y$, and every operator $T \in \mathcal{K}\left(Y, X^{* *}\right)$, there is a net $\left(S_{\gamma}\right) \subseteq \mathcal{K}(X, X)$ such that $\sup _{\gamma}\left\|S_{\gamma}^{* *} T\right\| \leq\|T\|$ and $\left.T^{*} S_{\gamma}^{*} \rightarrow T^{*}\right|_{X^{*}}$ in the strong operator topology.

Proof. (ii) $\Rightarrow$ (iii) $\Rightarrow$ (iv) are easy. 
(i) $\Rightarrow$ (ii). Let $Y$ be a Banach space and let $G \subseteq \mathcal{W}\left(Y, X^{* *}\right)$ be a finitedimensional subspace. As in the proof of Theorem 2.3 let

$$
K=\overline{\operatorname{conv}}\left\{T y: y \in B_{Y}, T \in B_{G}\right\} .
$$

Let

$$
\Gamma=\left\{(E, F): E \subseteq X^{* *}, \operatorname{dim} E<\infty, F \subseteq X^{*}, \operatorname{dim} F<\infty\right\},
$$

and order $\Gamma$ by inclusion. For $\gamma=(E, F) \in \Gamma$, let

$$
K_{\gamma}=\overline{\operatorname{conv}}\left(K \cup B_{E}\right) \subseteq B_{X^{* *}} .
$$

Then $K_{\gamma}$ is convex and weakly compact. As in the proof of (i) $\Rightarrow$ (ii) of Theorem 2.3, we find a reflexive Banach space $Z_{\gamma}$, a weakly compact norm one operator $J_{\gamma}: Z_{\gamma} \rightarrow X^{* *}$, and a linear isometry $\Psi_{\gamma}: G \rightarrow \mathcal{W}(Y, Z)$ such that $T=J_{\gamma} \circ \Psi_{\gamma}(T)$ for all $T \in G$.

Let

$$
\mathfrak{E}_{\gamma}=\left\{S^{* *} \circ J_{\gamma}: S \in \mathcal{K}(X, X)\right\} \subseteq \mathcal{K}\left(Z_{\gamma}, X\right),
$$

and let $\mathfrak{F}_{\gamma}=\operatorname{span}\left(\mathfrak{E}_{\gamma}, J_{\gamma}\right)$. Then we have

$$
\mathcal{F}\left(Z_{\gamma}, X\right) \subseteq \overline{\mathfrak{E}}_{\gamma} \subseteq \mathcal{K}\left(Z_{\gamma}, X\right)
$$

Lemma 3.5 tells us that $\mathfrak{E}_{\gamma}$ is an ideal in $\mathfrak{F}_{\gamma}$ with a Hahn-Banach extension operator $\Phi: \mathfrak{E}_{\gamma}^{*} \rightarrow \mathfrak{F}_{\gamma}^{*}$ satisfying $\Phi\left(x^{*} \otimes z\right)=x^{*} \otimes z$.

By Lemma 1.4 in [10], there is a net $\left(S_{\alpha}\right) \subseteq \mathcal{K}(X, X)$ with $\sup _{\alpha}\left\|S_{\alpha}^{* *} J_{\gamma}\right\|$ $\leq 1$ and $x^{*}\left(S_{\alpha}^{* *} J_{\gamma} z\right) \rightarrow x^{*}\left(J_{\gamma} z\right)$ for all $x^{*} \in X^{*}$ and all $z \in Z_{\gamma}$.

With $\gamma=(E, F) \in \Gamma$, let $x^{*} \in F$ and $x^{* *} \in E$. Then $x^{* *}=J_{\gamma} z$ for some $z \in Z_{\gamma}$. Thus

$$
x^{*}\left(S_{\alpha} J_{\gamma} z\right) \rightarrow x^{*}\left(J_{\gamma} z\right),
$$

which means that

$$
x^{* *}\left(S_{\alpha}^{*} x^{*}\right) \rightarrow x^{* *}\left(x^{*}\right) .
$$

Using the compactness of $S_{E}$ and $S_{F}$, choose $\alpha$ such that

$$
\left|x^{* *}\left(x^{*}\right)-x^{* *}\left(S_{\alpha}^{*} x^{*}\right)\right| \leq \frac{\left\|x^{*}\right\|\left\|x^{* *}\right\|}{\operatorname{dim} E+\operatorname{dim} F}
$$

for all $x^{*} \in F$ and all $x^{* *} \in E$, and let $S_{\gamma}=S_{\alpha}$. Then we get a net $\left(S_{\gamma}\right)_{\gamma \in \Gamma}$ such that $\sup _{\gamma}\left\|S_{\gamma}^{* *} T\right\| \leq\|T\|$ for all $T \in G$, and $S_{\gamma}^{*} \rightarrow I_{X^{*}}$ in the weak operator topology. By taking a new net, also denoted $\left(S_{\gamma}\right)$, from $\operatorname{conv}\left(S_{\gamma}\right)$, we may assume that $\sup _{\gamma}\left\|S_{\gamma}^{* *} T\right\| \leq\|T\|$ for all $T \in G$, and $S_{\gamma}^{*} \rightarrow I_{X^{*}}$ in the strong operator topology.

(iv) $\Rightarrow\left(\right.$ i). Let $\left\{x_{n}^{*}\right\}_{n=1}^{\infty} \subseteq X^{*},\left\{x_{n}^{* *}\right\}_{n=1}^{\infty} \subseteq X^{* *}$ with $\sum_{n=1}^{\infty}\left\|x_{n}^{* *}\right\|\left\|x_{n}^{*}\right\|$ $<\infty$ and $\sum_{n=1}^{\infty} x_{n}^{* *}\left(T^{*} x_{n}^{*}\right)=0$ for all $T \in \mathcal{K}(X, X)$. We may assume that $1 \geq\left\|x_{n}^{* *}\right\| \rightarrow 0$ and $\sum_{n=1}^{\infty}\left\|x_{n}^{*}\right\|<\infty$.

Let $K=\overline{\operatorname{conv}}\left( \pm x_{n}^{* *}\right) \subseteq B_{X^{* *}}$. We construct a reflexive Banach space $Z$ and a compact operator $J \in \mathcal{K}\left(Z, X^{* *}\right)$ with $\|J\| \leq 1$ as in Lemma 1.1 of [10]. 
Let $\left(S_{\gamma}\right)$ be a net in $\mathcal{K}(X, X)$ such that $\sup _{\gamma}\left\|S_{\gamma}^{* *} J\right\| \leq 1$ and $\left.J^{*} S_{\gamma}^{*} \rightarrow J^{*}\right|_{X^{*}}$ in the strong operator topology.

Choose positive numbers $\left\{a_{n}\right\}_{n=1}^{\infty}$ such that

$$
M=\sum_{n=1}^{\infty} a_{n}<\infty \quad \text { and } \quad\left\|x_{n}^{*}\right\| / a_{n} \rightarrow 0 .
$$

Let $z_{n} \in B_{Z}$ be such that $x_{n}^{* *}=J z_{n}$. For every $S \in \mathcal{K}(X, X)$, we have

$$
0=\sum_{n=1}^{\infty} x_{n}^{* *}\left(S^{*} x_{n}^{*}\right)=\sum_{n=1}^{\infty} z_{n}\left(J^{*} S^{*} x_{n}^{*}\right) .
$$

The set $\left\{x_{n}^{*} / a_{n}\right\}_{n=1}^{\infty}$ is relatively compact, so for every $\varepsilon>0$, there is some $S_{\gamma}$ such that

$$
\left\|J^{*} S_{\gamma}^{*} x_{n}^{*}-J^{*} x_{n}^{*}\right\|<\varepsilon a_{n} / M
$$

for all $n$. Thus we get

$$
\begin{aligned}
\left|\sum_{n=1}^{\infty} x_{n}^{* *}\left(x_{n}^{*}\right)\right| & =\left|\sum_{n=1}^{\infty} z_{n}\left(J^{*} x_{n}^{*}-J^{*} S_{\gamma}^{*} x_{n}^{*}\right)\right| \leq \sum_{n=1}^{\infty}\left\|z_{n}\right\| a_{n} \varepsilon / M \\
& \leq \frac{\varepsilon}{M} \sum_{n=1}^{\infty} a_{n}=\varepsilon .
\end{aligned}
$$

Thus $\sum_{n=1}^{\infty} x_{n}^{* *}\left(x_{n}^{*}\right)=0$, and $X^{*}$ has the compact approximation property with conjugate operators.

Corollary 3.7. Let $X$ be a Banach space. The following statements are equivalent:

(i) $X^{*}$ has the compact approximation property with conjugate operators.

(ii) For every Banach space $Y$ and every $T \in \mathcal{W}\left(X^{*}, Y\right)$, there is a net $\left(S_{\gamma}\right) \subseteq \mathcal{K}(X, X)$ with $\sup _{\gamma}\left\|T S_{\gamma}^{*}\right\| \leq\|T\|$ such that $T S_{\gamma}^{*} \rightarrow T$ in the strong operator topology.

Proof. This follows from (i) $\Leftrightarrow($ iii) of Theorem 3.6.

REMARK 3.2. Corollary 3.7 generalizes Corollary 3.3 of [13]. Grothendieck [7, proof of Theorem 15 on pp. 183-184] proved the implication (i) $\Rightarrow$ (ii) of Corollary 3.7 for the approximation property. This was made explicit by Reŭnov: see [17, Corollary 2 of Theorem 4].

In [1, Theorem 1] Casazza and Jarchow gave an example of a separable Banach space $X$ such that $X^{*}$ has the metric compact approximation property, while $X$ fails the metric compact approximation property.

From Corollary 4.7 and Example 1.2 in [12] it follows that the dual of Casazza and Jarchow's $X$ does not have the compact approximation property with conjugate operators. The next theorem gives a necessary and suf- 
ficient condition for the compact approximation property and the compact approximation property with conjugate operators to coincide.

Theorem 3.8. Let $X$ be a Banach space. The following statements are equivalent.

(i) $X^{*}$ has the compact approximation property with conjugate operators.

(ii) $\widehat{X}^{*}$ has the compact approximation property with conjugate operators for every equivalent renorming $\widehat{X}$ of $X$.

(iii) $X^{*}$ has the compact approximation property and $\mathcal{K}(X, \widehat{X})$ is an ideal in $\mathcal{K}\left(X, \widehat{X}^{* *}\right)$ for every equivalent renorming $\widehat{X}$ of $X$.

Proof. (i) $\Rightarrow$ (ii) is trivial, and (ii) $\Rightarrow$ (iii) is proved in [12, Corollary 4.7].

(iii) $\Rightarrow\left(\right.$ i). Let $\left\{x_{n}^{*}\right\} \subseteq X^{*},\left\{x_{n}^{* *}\right\} \subseteq X^{* *}$ such that $\sum_{n=1}^{\infty}\left\|x_{n}^{*}\right\|\left\|x_{n}^{* *}\right\|<\infty$ and $\sum_{n=1}^{\infty} x_{n}^{* *}\left(S^{*} x_{n}^{*}\right)=0$ for all $S \in \mathcal{K}(X, X)$. Without loss of generality, we may assume that $\sum_{n=1}^{\infty}\left\|x_{n}^{* *}\right\|<\infty$ and $\left\|x_{n}^{*}\right\| \rightarrow 0$.

We shall show that $\sum_{n=1}^{\infty} x_{n}^{* *}\left(T x_{n}^{*}\right)=0$ for all $T \in \mathcal{K}\left(X^{*}, X^{*}\right)$, which by assumption, and Theorem 3.1, implies that $\sum_{n=1}^{\infty} x_{n}^{* *}\left(x_{n}^{*}\right)=0$. A quick look at Theorem 3.3 then reveals that $X^{*}$ has the compact approximation property with conjugate operators.

By Theorem 4.4 in [14], there is a Hahn-Banach extension operator

$$
\Phi: \mathcal{K}(X, X)^{*} \rightarrow \mathcal{K}\left(X, X^{* *}\right)^{*}
$$

such that $\Phi\left(x^{*} \otimes x^{* *}\right)=x^{*} \otimes x^{* *}$ for all $x^{*} \in X^{*}$ and all $x^{* *} \in X^{* *}$. Let $P$ be the associated ideal projection.

Let $T \in \mathcal{K}\left(X^{*}, X^{*}\right)$ and let $U=\left.T^{*}\right|_{X} \in \mathcal{K}\left(X, X^{* *}\right)$. By Lemma 1.4 in [10], there is a net $\left(S_{\gamma}\right) \subseteq \mathcal{K}(X, X)$ such that

$$
\sup _{\gamma}\left\|S_{\gamma}\right\| \leq\left\|P^{*} U\right\| \leq\|U\| \leq\|T\|
$$

and

$$
x^{* *}\left(S_{\gamma}^{*} x^{*}\right) \underset{\gamma}{\rightarrow}\left(P\left(x^{*} \otimes x^{* *}\right)\right)(U)=x^{* *}\left(T x^{*}\right)
$$

for all $x^{*} \in X^{*}$ and all $x^{* *} \in X^{* *}$. By a well known convexity argument, we may assume that $S_{\gamma}^{*} \rightarrow T$ in the strong operator topology. Then $S_{\gamma}^{*} \rightarrow T$ uniformly on the relatively compact set $\left\{x_{n}^{*}\right\}_{n=1}^{\infty}$. Now it follows that

$$
\sum_{n=1}^{\infty} x_{n}^{* *}\left(T x_{n}^{*}\right)=0,
$$

as desired.

We shall say that a Banach space $X$ has the unique extension property if $\mathrm{B}\left(X, X^{* *}\right)$ consists of a single element (the natural embedding of $X^{*}$ into $\left.X^{* * *}\right)$. 
Theorem 3.9. Let $X$ be a Banach space. If $X$ has the unique extension property, then the following statements are equivalent:

(i) $X^{*}$ has the compact approximation property with conjugate operators.

(ii) $X^{*}$ has the compact approximation property, and $\mathcal{K}(Z, X)$ is an ideal in $\mathcal{K}\left(Z, X^{* *}\right)$ for all Banach spaces $Z$.

Proof. The proof is similar to that of Theorem 3.8. Note that if $T \in$ $\mathcal{K}\left(X^{*}, X^{*}\right)$, then by Theorem 2.3 in [13] there is a net $\left(S_{\gamma}\right) \subseteq \mathcal{K}(X, X)$, with $\sup _{\gamma}\left\|S_{\gamma}\right\| \leq\|T\|$, such that

$$
S_{\gamma}^{*} x^{*} \rightarrow\left(\left.T^{*}\right|_{X}\right)^{*} x^{*}=T x^{*}
$$

for all $x^{*} \in X^{*}$. (We have $\left.T^{*}\right|_{X} \in \mathcal{K}\left(X, X^{* *}\right)$.)

\section{References}

[1] P. G. Casazza and H. Jarchow, Self-induced compactness in Banach spaces, Proc. Roy. Soc. Edinburgh Sect. A 126 (1996), 355-362.

[2] C.-M. Cho and W. B. Johnson, A characterization of subspaces $X$ of $\ell_{p}$ for which $K(X)$ is an $M$-ideal in $L(X)$, Proc. Amer. Math. Soc. 93 (1985), 466-470.

[3] W. J. Davis, T. Figiel, W. B. Johnson, and A. Pełczyński, Factoring weakly compact operators, J. Funct. Anal. 17 (1974), 311-327.

[4] P. G. Dixon, Left approximate identities in algebras of compact operators on Banach spaces, Proc. Roy. Soc. Edinburgh Sect. A 104 (1986), 169-175.

[5] H. Fakhoury, Sélections linéaires associées au théorème de Hahn-Banach, J. Funct. Anal. 11 (1972), 436-452.

[6] M. Feder and P. Saphar, Spaces of compact operators and their dual spaces, Israel J. Math. 21 (1975), 38-49.

[7] A. Grothendieck, Produits tensoriels topologiques et espaces nucléaires, Mem. Amer. Math. Soc. 16 (1955).

[8] N. J. Kalton, Locally complemented subspaces and $\mathcal{L}_{p}$-spaces for $0<p<1$, Math. Nachr. 115 (1984), 71-97.

[9] A. Lima, The metric approximation property, norm-one projections and intersection properties of balls, Israel J. Math. 84 (1993), 451-475.

[10] A. Lima, O. Nygaard, and E. Oja, Isometric factorization of weakly compact operators and the approximation property, ibid. 119 (2000), 325-348.

[11] A. Lima and E. Oja, Ideals of finite rank operators, intersection properties of balls, and the approximation property, Studia Math. 133 (1999), 175-186.

[12] - - - Ideals of compact operators, J. Austral. Math. Soc., to appear.

[13] - - - Ideals of operators, approximability in the strong operator topology, and the approximation property, submitted.

[14] V. Lima, Approximation properties for dual spaces, Math. Scand., to appear.

[15] J. Lindenstrauss and L. Tzafriri, Classical Banach Spaces I, Springer, Berlin, 1977.

[16] -, 一, Classical Banach Spaces II, Springer, Berlin, 1979.

[17] O. I. Reĭnov, Operators of RN type in Banach spaces, Sibirsk. Mat. Zh. 19 (1978), 857-865, 955 (in Russian); English transl.: Siberian Math. J. 19 (1978), 606-612.

[18] A. Szankowski, Subspaces without the approximation property, Israel J. Math. 30 (1978), 123-129. 
[19] G. Willis, The compact approximation property does not imply the approximation property, Studia Math. 103 (1992), 99-108.

Department of Mathematics

Agder University College

Serviceboks 422

4604 Kristiansand, Norway

E-mail: Vegard.Lima@hia.no

Asvald.Lima@hia.no

Olav.Nygaard@hia.no 